\title{
18ß-Glycyrrhetinic Acid Suppresses Allergic Airway Inflammation through NF-KB and Nrf2/HO-1 Signaling Pathways in Asthma Mice
}

jianming liu ( $\square$ liujianming390@163.com )

Nanchang University

Yanqi Xu

Jiangxi Medical College

Minyu Yan

Nanchang University

Yingjie Yu

Nanchang University

Yongmei Guo

Jiangxi Medical College

\section{Research Article}

Keywords: $18 \beta$-Glycyrrhetinic acid (18ß-GA), bioactive component, Glycyrrhizae Radix, anti-inflammatory and antioxidant agent

Posted Date: August 10th, 2021

DOl: https://doi.org/10.21203/rs.3.rs-751481/v1

License: (1) This work is licensed under a Creative Commons Attribution 4.0 International License.

Read Full License

Version of Record: A version of this preprint was published at Scientific Reports on February 24th, 2022.

See the published version at https://doi.org/10.1038/s41598-022-06455-6. 


\section{Abstract}

$18 \beta$-Glycyrrhetinic acid (18ß-GA), the main bioactive component of Glycyrrhizae Radix, is considered a promising anti-inflammatory and antioxidant agent. Here, we evaluated the anti-inflammatory and antioxidant effects of 18ß-GA in an ovalbumin (OVA)-induced asthma mouse model, and examined the role of NF-kB and Nrf2/HO-1 signaling pathways. The histopathological changes of lung tissue in mouse were assessed by histochemical staining and counting of inflammatory cells. The levels of IgE and inflammatory cytokines in the bronchoalveolar lavage fluid of mice were detected by ELISA. In OVAinduced asthmatic mice, $18 \beta-G A$ treatment can significantly improve lung function and reduce lung inflammation including infiltration of inflammatory cells. In addition, 18ß-GA reduced the OVA-induced NF-KB phosphorylation in lungs of mice while increasing the expression of Nrf2 and HO-1. These results indicate that 18ß-GA protects OVA-induced allergic inflammation of airway by inhibiting phosphorylation of NF-KB and enhancing the Nrf2/HO-1 pathway, and serves as a potential treatment option for allergic inflammation of airway.

\section{Introduction}

The chronic inflammatory disease asthma affects around 300 million people worldwide ${ }^{1}$. Asthma is a lung disorder with characteristics of inflammation and narrowing of the respiratory tracts, which involves oxidative stresses. Increased productions of pro-inflammatory cytokines, reactive oxygen species (ROS), and growth factors are key features of asthma ${ }^{2,3}$. Existing antioxidant systems can remove superoxide dismutase (ROS), with catalase, superoxide dismutase (SODs), and glutathione peroxidase (GSHPx) being the common antioxidant enzymes ${ }^{4}$. Heme oxygenase $1(\mathrm{HO}-1)$ is a nuclear factor erythroid 2related factor2 (Nrf2)-regulated antioxidant that promotes the production of antioxidant molecules ${ }^{5}$. The HO-1 expression reduces nuclear factor kappa B (NF-KB) level, thereby inhibiting the inducible nitric oxide synthase (iNOS), and showing anti-asthmatic effects through inhibition on ROS production and inflammatory response ${ }^{6}$. Furthermore, a growing number of evidence suggest that NF-KB, being a multicellular transcription factor, regulates immune and inflammatory responses through modulating cytokine productions ${ }^{7,8}$. It is reported that in allergic asthma, the NF-KB pathway is activated, and the suppression of NF-KB signaling can relieve ovalbumin (OVA)-induced asthma ${ }^{9,10}$. Therefore, in the treatment of asthma, in addition to alleviating airway inflammation and obstruction, we should also consider suppressing oxidative stresses.

Currently, there are few anti-asthmatic drugs with stable curative effects and few side effects available to patients. Long-acting beta-agonist and inhaled corticosteroids are considered the most common therapeutic options to treat asthma. Although they reduce airway inflammation and attenuate respiratory symptoms, have poor responses to corticosteroid-based medications are encountered in some patients, with serious adverse effects in some cases ${ }^{11,12}$. The number of reported asthma cases is increasing rapidly, and alternative therapeutic approaches are urgently needed. In view of the production of ROS and 
other oxidants in asthma, we suggest that therapeutics targeting redox stresses and signaling molecules may effectively treat asthma ${ }^{13,14}$.

Glycyrrhizic acid is the triterpene component extracted from licorice root, and in China and other Asian countries, it has been long used for the treatment of chronic hepatitis $\mathrm{B}^{15}$. The glycyrrhizin metabolized by intestinal flora, and its product is $18 \beta$-Glycyrrhetinic acid $(18 \beta-G A)^{16}$. 18ß-GA showed anti-ulcer and anti-inflammation effects ${ }^{17}$, and its chemical structure is depicted in Fig. 1. Although 18ß-GA is related to the immunoregulatory function of allergic diseases and the improvement of allergic asthma ${ }^{18}$, the pathophysiological role of $18 \beta-G A$ in allergic lung inflammation remains unclear. Its therapeutic effect on allergic asthma and the possible mechanisms need to be further investigated. The purpose of the study is to investigate the effects of $18 \beta-G A$ in treating chronic allergic asthma and its possible mechanisms using a mouse model of allergic asthma.

\section{Results}

\section{$18 \beta-G A$ reduces airway responsiveness of mice to methacholine $(\mathrm{MCH})$ after allergen challenge.}

In order to investigate the effects of $18 \beta-G A$ on asthma, OVA was used to challenge mice intranasally to establish the mouse asthmatic model to evaluate the airway response to inhaled $\mathrm{MCH}$ (The procedure of the experiment is presented in Fig. 2). The changes in lung resistance ( $\mathrm{RL})$ and lung dynamic compliance (Cdyn) were measured by increasing inhaled $\mathrm{MCH}$. Compared with normal mice, the RL increased in the asthmatic mouse groups, and the RL also increased with the increase of inhaled $\mathrm{MCH}$. However, the RL of asthmatic mice treated with Mon decreased significantly (Fig. 3a). The RL of asthmatic mice treated with $18 \beta$-GA also showed a downward trend, and the decrease was more obvious as the concentration of $18 \beta-$ GA increased. In addition, compared with normal mice, the Cdyn in the asthmatic mouse groups decreased significantly, but Cydn increased after treatment with Mon or $18 \beta-G A$, and the increase in the Cydn was more obvious with the increase of the concentration of 18ß-GA (Fig. 3b).

\section{$18 \beta-G A$ alleviates lung pathological changes.}

H\&E and periodate Schiff (PAS) staining were used to detect pathological changes in the lungs. There were many infiltrated inflammatory cells in the asthma group, showing obvious vascular edema, while treatment of Mon or 18ß-GA can significantly reduce the number of infiltrated inflammatory cells $(p=$ 0.016 and $p=0.009$, respectively)(Figs. $4 \mathrm{a}, \mathrm{b}$ ). The results of PAS staining showed that compared with the normal mice, the asthma mice had significantly increased mucus secretion and number of goblet cells, while both montelukast (Mon) and $18 \beta-G A(40)$ could reduce the amount of mucus and the number of goblet cells $(p=0.037$ and $p=0.039$, respectively) (Fig. 4c, d). 


\section{8ß-GA decreased IL-4, IL-5, IL-13, TNF- $a$ and IgE levels while increased IFN- $\gamma$ level.}

Compared with the normal mice, the asthma mice had significantly increased total inflammatory cell counts, eosinophils, neutrophils and lymphocytes (Fig. 5a). After treatment with Mon or 18ß-GA, inflammatory cell counts decreased, and the decrease was more obvious with the increase of dose. Further analysis on IL-5, IL-13, and IFN-y levels showed that compared with the normal control, the asthma group had higher IL-5, IL-13 and TNF-a levels and lower IFN- $\gamma$ levels. Treatment with 18ß-GA decreased the levels of IL-5, IL-13 and TNF- $\mathrm{a}(p=0,008 p=0.000$ and $p=0.006$, respectively) while increased the level of IFN-Y $(p=0.007)$ (Fig. 5b). Although the OVA-specific IgE level and the total IgE level elevated significantly ( $p=0.000$ and $p=0.000$, respectively) in the asthma group, they decreased significantly after treatment with $18 \beta-\mathrm{GA}(p=0.000$ and $p=0.000$, respectively) (Fig. $5 \mathrm{c}$ ).

\section{$18 \beta-G A$ reduced oxidative stress and increased the amount of antioxidant enzymes.}

In mice with OVA-induced asthma, the lung levels of ROS, MDA increased and the lung levels of T-AOC, SOD, CAT and GSH-Px decreased. Compared with the asthma group without treatment, 18ß-GA treatment groups had significantly lower levels of $\operatorname{ROS}$ and $\operatorname{MDA}(p=0.000$ and $p=0.000$, respectively), and higher levels of T-AOC, SOD, CAT, and GSH-Px ( $p=0.006, p=0.008, p=0.024$ and $p=0.031$, respectively), protecting the lungs from oxidative damage (Fig. 6 ).

\section{$18 \beta-G A$ inhibits allergic inflammation of airway through the NF-KB and Nrf2/HO-1 pathways.}

Previous results suggest that $18 \beta-G A$ can improve lung function in mice with allergic airway inflammation induced by OVA. The effect of 18ß-GA on the protein levels of p-NF-KB, Nrf2 and HO-1 were examined in mice treated with Mon and 18ß-GA. The results indicated that p-NF-KB, Nrf2 and HO-1 protein expressions were up-regulated in the asthma group, while $\mathrm{p}-\mathrm{NF}-\mathrm{kB}$ expression were down-regulated, while nuclear Nrf2 and HO-1 expression were further up-regulated after 18ß-GA(40) treatments $(p=0.014$ and $p$ $=0.015$, respectively) (Fig. 7).

\section{Discussion}

Asthma is an oxidative stress disorder characterized by airway inflammation and hyperresponsiveness ${ }^{19,20}$. Increased number of eosinophils is considered to be the main biomarker of asthma ${ }^{21}$. Cytokines contribute to the gathering of eosinophils within the lesions of the lungs ${ }^{22,23}$, facilitate the activation, maturation, and accumulation of eosinophils, and finally lead to IgE production, hyperproduction of mucus, and airway inflammation ${ }^{24,25}$. The regulation of pro-inflammatory cytokines is 
vital in the control of asthma. In the current study, 18ß-GA showed protective effects in mice with OVAinduced allergic airway inflammation. The $18 \beta-G A$ treatment significantly improved lung function and reduced lung inflammation including inflammatory cell infiltration. The treatment of $18 \beta-G A$ reduced IL-5, IL-13, TNF- $a$, OVA-specific IgE level and total IgE level while increased IFN- $\gamma$ level, thereby helping to reduce the influx of leukocytes, goblet cell metaplasia, and airway hyperresponsiveness, as shown by lung histopathological analysis. In addition, levels of ROS, MDA, T-AOC were examined in the current study. The 18ß-GA treatment group reduced the levels of ROS and MDA significantly and restored the levels of T-AOC and SOD, CAT and GSH-Px. MDA is a ROS metabolite and its level reflects protein denaturation, lipid peroxidation, and impaired endothelial cell integrity ${ }^{26,27}$. During an asthma attack, the activity of peroxides, MDA, conjugated dienes increased and activity of SOD decreased significantly $28,29$. SOD, an important endogenous antioxidative enzyme in lung tissue, was restored by $18 \beta-G A$ treatment, leading to relief of the oxidative damage and alleviation of asthma in our study ${ }^{30}$.

The signal transduction of inflammation and oxidative stress were examined to understand the antiinflammatory and antioxidant effects of $18 \beta-G A$. NF- $K B$ is one of the main contributors to the inflammatory pathway, and NF-KB p65 phosphorylation and IкB kinase activation are characterized in respiratory tract inflammation ${ }^{31,32}$. Consistently, our study found that NF-KB activation was promoted by OVA exposure in asthmatic mice. In addition, NF-KB activation is related to the mechanism of ROSmediated oxidative stress ${ }^{33}$. Here, the OVA treatment-induced asthma, activated the NF-KB pathway and overproduced ROS in lung tissues, which could be a result of ROS and NF-KB crosstalk. The anti-oxidative pathway Nrf2/HO-1 is vital in cellular defense. It has shown that $\mathrm{HO}-1$ can relieve airway oxidative stresses, mucus hypersecretion and inflammation effectively in asthma ${ }^{34}$. Nrf2 is the key factor for HO-1 transcription, and in physiological conditions, it is mainly located in the cytoplasm in association with the inhibitory protein Keap1. At stimulation, Nrf2 is translocated to the nucleus, where it realizes the antioxidant effect through binding to the antioxidant response element (ARE) and activating the transcription of the HO-1 gene. In this study, 18ß-GA increased the nuclear expression of Nrf2 and the lung expression of HO-1 in the OVA-treated mice significantly. The results indicate that $18 \beta-G A$ reduces oxidative damage and airway inflammation. through the promotion of the Nrf2/HO-1 pathway.

In conclusion, treatment of 18$\beta-$-GA on OVA-induced allergic airway inflammation can suppress eosinophilia, reduce the IgE level, pro-inflammatory cytokines level, and alleviate airway hyperresponsiveness, as evidenced by alleviation in respiratory tract inflammation and less mucus production. The anti-asthmatic potency of $18 \beta-\mathrm{GA}$ is related to the inhibition of Nrf2/HO-1 pathway activation and NF-KB phosphorylation. The study indicates that 18ß-GA may be a promising therapy to suppress the development of asthma.

\section{Methods}

Mice. 
Female specific pathogen-free (SPF) inbred BALB/c mice(6 weeks, 20-25 g)were obtained from Hunan SJA Laboratory Animal Co.,Ltd (Changsha, China; license \#: SCXK (Xiang) 2013-0006. The animals were maintained in an SPF animal house, with a regular $12 \mathrm{~h} / 12 \mathrm{~h}$ light/dark cycle, relative humidity of 40$70 \%$, and an average temperature of $25^{\circ} \mathrm{C}$.

\section{Ethics committees.}

The study was carried out in compliance with the ARRIVE guidelines. The study was approved by the Animal Care and Use Committee of the Medical College of Jiangxi Medical College. Experiments were performed in accordance with the guidelines of the United States National Institutes of Health $(\mathrm{NIH})$ regarding the care and use of animals for experimental procedures.

\section{Chemicals and reagents.}

OVA (CAS NO. 9006-59-1, Biotechnology grade), montelukast (CAS N0.151767-02-1, LC\&T, purity $\geq 98 \%$ ) and 18ß-GA(CAS NO. 471-53-4, purity $\geq 98 \%$ ) were obtained from Macklin Biochemical Co., Ltd. (Shanghai, China).

\section{Asthmatic model induced by OVA and drug administration.}

The mouse model of asthma was induced by intraperitoneal injection of OVA $(20 \mu \mathrm{g})$, which was mixed with aluminum hydroxide ( $2 \mathrm{mg}$ ) on day 1 and day 14 . From day 18 to day 23, the mice received oral gavage of Mon $(10 \mathrm{mg} / \mathrm{kg})$ and 18ß-GA $(10,20$, and $40 \mathrm{mg} / \mathrm{kg})$. The control group received saline of the same amount. On day 21 to day 23, 1\% OVA aerosol in PBS was given to the sensitized mice for 20 minutes. On day 24, whole-body plethysmography (Buxco Electronics, Troy, NY) was used to evaluate airway hyperresponsiveness ${ }^{35}$. There were 6 groups of mice, with 5 mice in each group $(n=5)$ : Control (normal control), Asthma (OVA sensitization), Asthma + Mon (OVA sensitization and Mon administration), Asthma + 18ß-GA 10, 20, and 40 (OVA sensitization and 18ß-GA gavage of 10, 20, and $40 \mathrm{mg} / \mathrm{kg}$, respectively). The procedure of the experiment is presented in Fig. 2.

\section{Measurement of airway hyperresponsiveness to inhaled $\mathrm{MCH}$.}

The airway hyperresponsiveness was measured $24 \mathrm{~h}$ after the last OVA challenge. The experiment was carried out when the mice were anesthetized with sodium pentobarbital (100 mg/kg, i.p.): the mouse trachea was incised, then intubated and placed in the whole body plethysmograph, which was connected to the ventilator. Then, when the mice were exposed to increasing concentrations $(0,3.125,6.25$, and 12.5 $\mathrm{mg} / \mathrm{mL}$ ) of $\mathrm{MCH}$, record the $\mathrm{RL}$ and Cdyn. 


\section{Lung histopathology.}

Lung tissues were fixed with $10 \%$ formalin, paraffin-embedded, and cut into $4 \mu \mathrm{m}$ sections, then stained with H\&E to assess infiltration of inflammatory cells, or stained with PAS to assess the mucus production of goblet cells. The inflammation of the lung tissue and mucus production was quantitatively analyzed by an image analyzer.

\section{Assessment of bronchoalveolar lavage fluid and serum.}

The mice were anesthetized with sodium pentobarbital (100 mg/ $\mathrm{kg}$, i.p.) and the lungs were exposed by thoracotomy. The mice were intubated and intratracheally instilled with PBS twice before the bronchoalveolar lavage fluid (BALF) collection. After these procedures were completed, the mice were sacrificed by cervical dislocation. The lavage solution was then centrifuged at $4^{\circ} \mathrm{C}$ and $500 \mathrm{~g}$ for 10 minutes. Differential cell counts were performed and stained with Diff-Quik (Solarbio, Beijing). The cytokines in the supernatant were detected and the cell precipitation was resuspended. The type and number of leukocytes were calculated by a cell counter. The levels of IL-5, IL-13, IFN- $\gamma$, TNF- $\alpha$ and IgE were determined by ELISA kits (Nanjing Jiancheng Bioengineering Institute, China).

\section{Evaluation of lung oxidative stress.}

Samples of lung tissues was cut up and homogenized with RIPA cold lysis buffer for 3 minutes. After centrifugation (12000 g, at $4^{\circ} \mathrm{C}$ for $10 \mathrm{~min}$ ), lung ROS, T-AOC, MDA, CAT, SOD and GSH-Px were measured on a 96 well plate by a commercially available kit (Beyotime Institute of Biotechnology). The activities of ROS, T-AOC, MDA, CAT, SOD, and GSH-Px were detected by the ROS, T-AOC, MDA, SOD, CAT, and GSH-Px assay kits, then the absorbance at $485 \mathrm{~nm}, 593 \mathrm{~nm}, 532 \mathrm{~nm}, 450 \mathrm{~nm}, 520 \mathrm{~nm}$ and $340 \mathrm{~nm}$ was measured by a microplate reader, respectively.

\section{Western Blotting.}

The lung tissue and lysate containing protease inhibitor were homogenized, then centrifuged at $4^{\circ} \mathrm{C}$ at 12 $000 \mathrm{~g}$ to retain the supernatant. The $25 \mu \mathrm{L}$ of 15 -fold diluted supernatant was used for protein quantification. The loading buffer was added, and the remaining supernatant was separated by SDSPAGE, and then transferred to PVDF membrane. Then sealed at room temperature for 2 hours and incubated with the primary antibodies $(1: 1000)$ overnight at $4{ }^{\circ} \mathrm{C}$. On the second day, the second antibody (1:15 000) was applied for 1 hour, and the ECL kit was used for photodetection. The primary antibodies including anti-HO-1 Abs, anti-NF-KB Abs, anti-phospho anti-Nrf2 Abs, and NF-KB Abs that were used in the study were purchased from Proteintech Technology Inc. 


\section{Statistical analysis.}

Data are expressed as mean \pm standard deviation (SD). The analysis used one-way ANOVA with Tukey post hoc test. Graphs are generated using Graphpad prism 8 . The statistically significances were set as the following: $p<0.05, p<0.01$ and $p<0.001$.

\section{Declarations}

\section{Acknowledgments}

This work was supported by funding from the Department of Science and Technology Program Funds of Jiangxi Province, China (No. 20151BAB205085). In addition, the authors acknowledge Jiawei Liu (School of Pharmacy, Nanchang University) for his assistance in the study.

\section{Author contributions}

Jianming Liu, Yanqi Xu and Yongmei Guo performed the experiments. Jianming Liu, Minyu Yan and Yingjie Yu wrote the manuscript. Jianming Liu and Yanqi Xu designed experiments.

\section{Competing interests}

The authors declare no competing interests.

\section{References}

1. Huang, C. et al. Impact of nasal symptoms on the evaluation of asthma control., $96, \mathrm{e} 6147$ https://doi.org/10.1097/MD.0000000000006147 (2017).

2. Gauthier, M., Ray, A. \& Wenzel, S. E. Evolving Concepts of Asthma. Am J Respir Crit Care Med, 192, 660-668 https://doi.org/.10.1164/rccm.201504-0763PP( (2015).

3. Akiki, Z. et al. Serum cytokine profiles as predictors of asthma control in adults from the EGEA study. Respir Med, 125, 57-64 https://doi.org/10.1016/j.rmed.2017.03.002 (2017).

4. Dal, S. et al. Featured Article: Oxidative stress status and liver tissue defenses in diabetic rats during intensive subcutaneous insulin therapy.Exp Biol Med (Maywood)241:184 92.https://doi.org/10.1177/1535370215603837(2016).

5. Liu, Q., Gao, Y. \& Ci, X. Role of Nrf2 and Its Activators in Respiratory Diseases. Oxid Med Cell Longev, 2019, 7090534 https://doi.org/10.1155/2019/7090534 (2019).

6. Zhang, Z. H. et al. Mesenchymal stem cells increase expression of heme oxygenase-1 leading to antiinflammatory activity in treatment of acute liver failure. Stem Cell Res Ther, 8, 70 https://doi.org/10.1186/s13287-017-0524-3 (2017). 
7. Adcock, I. M., Caramori, G. \& Chung, K. F. New targets for drug development in asthma., 372, 10731087 https://doi.org/.10.1016/S0140-6736(08)61449-X (2008).

8. Yi, L. et al. Formononetin Attenuates Airway In fl ammation and Oxidative Stress in Murine Allergic Asthma. Front Pharmacol, 11, 533841 https://doi.org/10.3389/fphar.2020.533841 (2020).

9. Li, H. Y. et al. Propofol Attenuates Airway Inflammation in a Mast Cell-Dependent Mouse Model of Allergic Asthma by Inhibiting the Toll-like Receptor 4/Reactive Oxygen Species/Nuclear Factor kappaB Signaling Pathway. Inflammation, 41, 914-923 https://doi.org/10.1007/s10753-018-0746-2 (2018).

10. Wang, C. et al. Aloperine suppresses allergic airway inflammation through NF-kappaB, MAPK, and Nrf2/HO-1 signaling pathways in mice.Int Immunopharmaco/65:571 79.https://doi.org/10.1016/j.intimp.2018.11.003(2018).

11. Wang, Y. et al. Formononetin alleviates hepatic steatosis by facilitating TFEB-mediated lysosome biogenesis and lipophagy.J Nutr Biochem73:108214.https://doi.org/10.1016/j.jnutbio.2019.07.005(2019).

12. Aalbers, R., Vogelmeier, C. \& Kuna, P. Achieving asthma control with ICS/LABA: A review of strategies for asthma management and prevention. Respir Med, 111, 1-7 https://doi.org/10.1016/j.rmed.2015.11.002 (2016).

13. Kim, J. H. et al. Effects of the aqueous extract of Epimedii Herba on the induction of oral tolerance in mice. Biol. Pharm. Bull, 25, 1000-1005 https://doi.org/10.1248/bpb.25.1000 (2002).

14. Garcia-Vasquez, C., Fernandez-Acenero, M. J., Garcia Gomez-Heras, S. \& Pastor, C. Fibrin patch influences the expression of hypoxia-inducible factor-1alpha and nuclear factor-kappaBp65 factors on ischemic intestinal anastomosis. Exp Biol Med (Maywood)243:80308.https://doi.org/10.1177/1535370218777216(2018).

15. Akao, T. et al. Hydrolysis of glycyrrhizin to 18 beta-glycyrrhetyl monoglucuronide by lysosomal betaD-glucuronidase of animal livers.Biochem Pharmaco/41:1025-9.https://doi.org/10.1016/00062952(91)90210-v(1991).

16. Huang, Q. C. et al. Can active components of licorice, glycyrrhizin and glycyrrhetinic acid, lick rheumatoid arthritis? Oncotarget, 7, 1193-1202 https://doi.org/10.18632/oncotarget.6200 (2016).

17. Yang, Y. et al. Synthesis, anti-microbial and anti-inflammatory activities of 18beta-glycyrrhetinic acid derivatives. Bioorg Chem, 101, 103985 https://doi.org/10.1016/j.bioorg.2020.103985 (2020).

18. Kim, S. H., Hong, J. H., Lee, J. E. \& Lee, Y. C. 18beta-Glycyrrhetinic acid, the major bioactive component of Glycyrrhizae Radix, attenuates airway inflammation by modulating Th2 cytokines, GATA-3, STAT6, and Foxp3 transcription factors in an asthmatic mouse model.Environ Toxicol Pharmaco/52:99-113.https://doi.org/10.1016/j.etap.2017.03.011(2017).

19. Mishra, V., Banga, J. \& Silveyra, P. Oxidative stress and cellular pathways of asthma and inflammation: Therapeutic strategies and pharmacological targets. Pharmacol. Ther181,16982https://doi.org/10.1016/j.pharmthera.2017.08.011(2018). 
20. Hu, D. Role of Anti-inflammatory Cytokines IL-35 and IL-37 in Asthma. Inflammation, 40, 697-707 https://doi.org/10.1007/s10753-016-0480-6 (2017).

21. Shrestha, P. N. et al. Stability of peripheral blood immune markers in patients with asthma. Allergy Asthma Clin Immunol, 15, https://doi.org/:30.10.1186/s13223-019-0343-4 (2019).

22. Lambrecht, B. N., Hammad, H. \& Fahy, J. V. The Cytokines of Asthma., 50, 975-991 https://doi.org/10.1016/j.immuni.2019.03.018 (2019).

23. Barnes, P. J. Targeting cytokines to treat asthma and chronic obstructive pulmonary disease. Nat Rev Immunol, 18, 454-466 https://doi.org/10.1038/s41577-018-0006-6 (2018).

24. Montero-Perez, O., Contreras-Rey, M. B. \& Sanchez-Gomez, E. Effectiveness and safety of mepolizumab in severe refractory eosinophilic asthma: results in clinical practice. Drugs Context, $\mathbf{8}$, 212584 https://doi.org/10.7573/dic.212584 (2019).

25. Xu, M. H. et al. Association of interleukin-18 and asthma. Inflammation, 40, 324-327 https://doi.org/10.1007/s10753-016-0467-3 (2017).

26. Fang, W. T., Li, H. J. \& Zhou, L. S. Protective effects of prostaglandin E1 on human umbilical vein endothelial cell injury induced by hydrogen peroxide. Acta Pharmacol Sin, 31, 485-492 https://doi.org/10.1038/aps.2010.23 (2010).

27. Zhao, X. et al. Salidroside inhibits endogenous hydrogen peroxide induced cytotoxicity of endothelial cells. Biol Pharm Bull, 36, 1773 https://doi.org/8.10.1248/bpb.b13-00406 (2013).

28. Kianian, F. et al. Nephroprotection through Modifying the Apoptotic TNF-alpha/ERK1/2/Bax Signaling Pathway and Oxidative Stress by Long-term Sodium Hydrosulfide Administration in Ovalbumin-induced Chronic Asthma. Immunol Invest, 1-28 https://doi.org/10.1080/08820139.2020.1858860 (2020).

29. Seo, Y. S. et al. Codonopsis lanceolata attenuates allergic lung inflammation by inhibiting Th2 cell activation and augmenting mitochondrial ROS dismutase (SOD2) expression. sci.rep 9:2312. https://doi.org/10.1038/s41598-019-38782-6(2019).

30. Randall, M. J. et al. Anti-Inflammatory Effects of Levalbuterol-Induced 11ß-Hydroxysteroid Dehydrogenase Type 1 Activity in Airway Epithelial Cells. Front Endocrinol, 236, 1-13 https://doi.org/10.3389/fendo.2014.00236 (2015).

31. Papi, A. et al. Rhinovirus infection causes steroid resistance in airway epithelium through nuclear factor kappaB and c-Jun N-terminal kinase activation.J Allergy Clin Immuno/132:107585e6.https://doi.org/10.1016/j.jaci.2013.05.028(2013).

32. Ye, P., Yang, X. L., Chen, X. \& Shi, C. Hyperoside attenuates OVA-induced allergic airway inflammation by activating Nrf2. Int Immunopharmacol, 44, 168-173 https://doi.org/10.1016/j.intimp.2017.01.003 (2017).

33. Morgan, M. J. \& Liu, Z. G. Crosstalk of reactive oxygen species and NF-kappaB signaling. Cell Res, 21, 103-115 https://doi.org/10.1038/cr.2010.178 (2011).

34. Cheng, H. T. et al. Ferritin heavy chain mediates the protective effect of heme oxygenase-1 against oxidative stress.Biochim Biophys Acta1850:2506- 
17.https://doi.org/10.1016/j.bbagen.2015.09.018(2015).

35. Jung, T. Y. et al. Scrophularia koraiensis Nakai Attenuates Allergic Airway Inflammation via Suppression of NF-kappaB and Enhancement of Nrf2/HO-1 Signaling. Antioxidants (Basel), 9 (2), 99 https://doi.org/10.3390/antiox9020099 (2020).

\section{Figures}

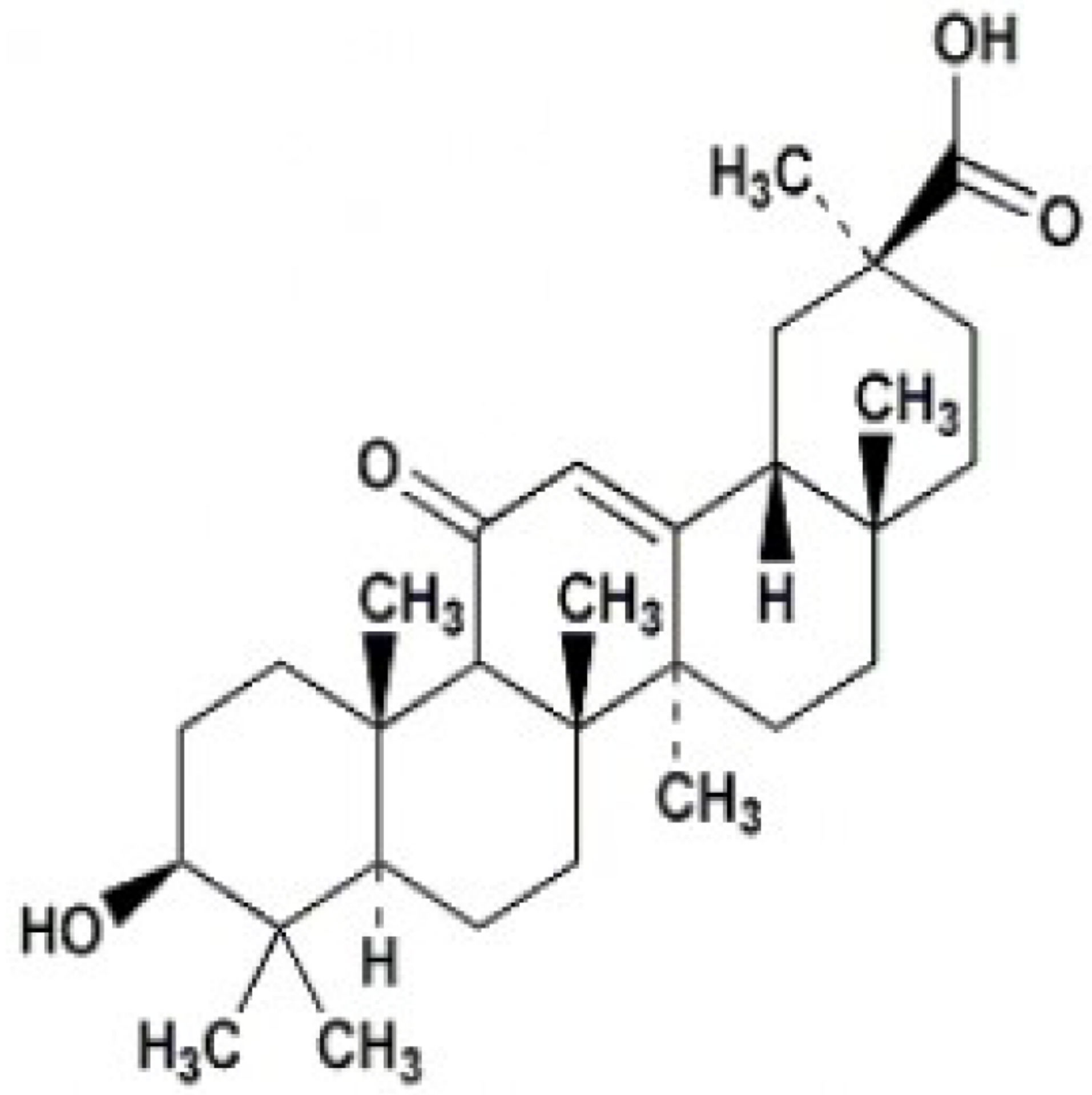

Figure 1 


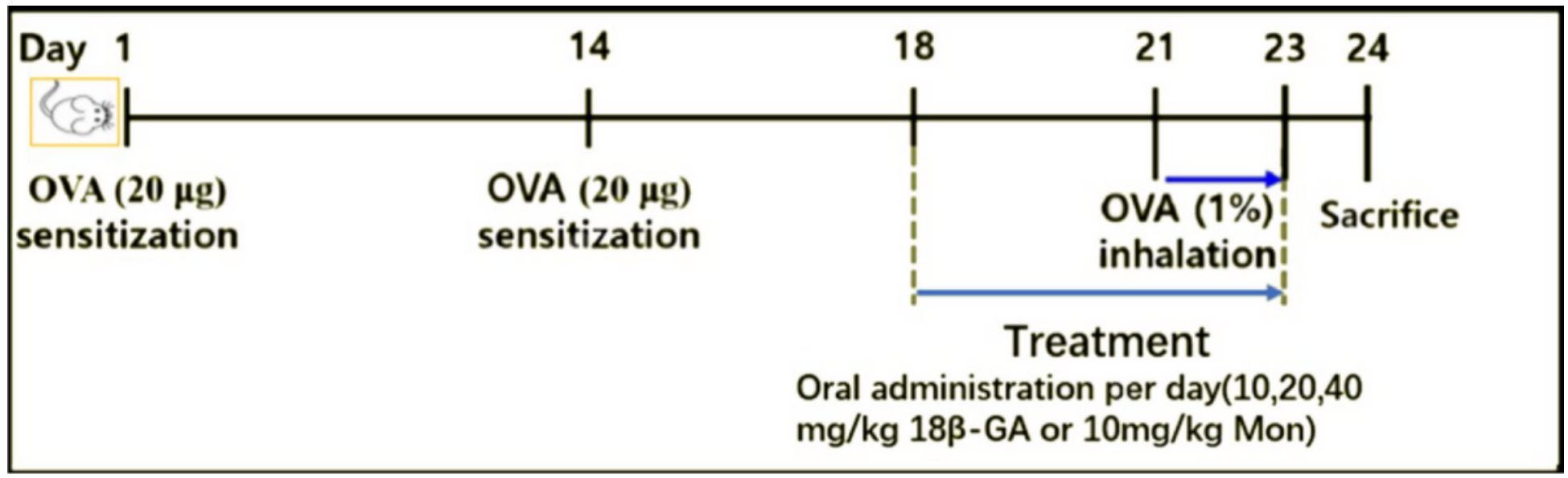

Figure 2

Asthmatic model and treatment protocol

a

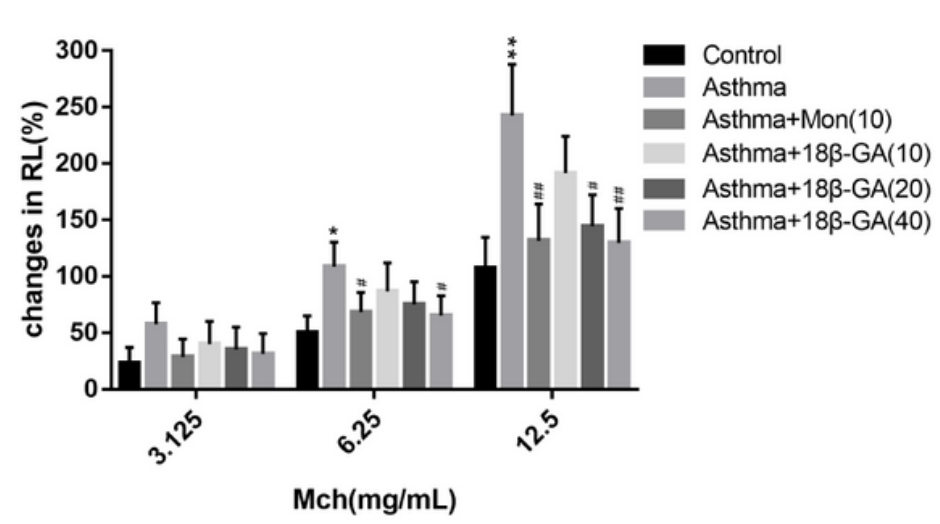

b

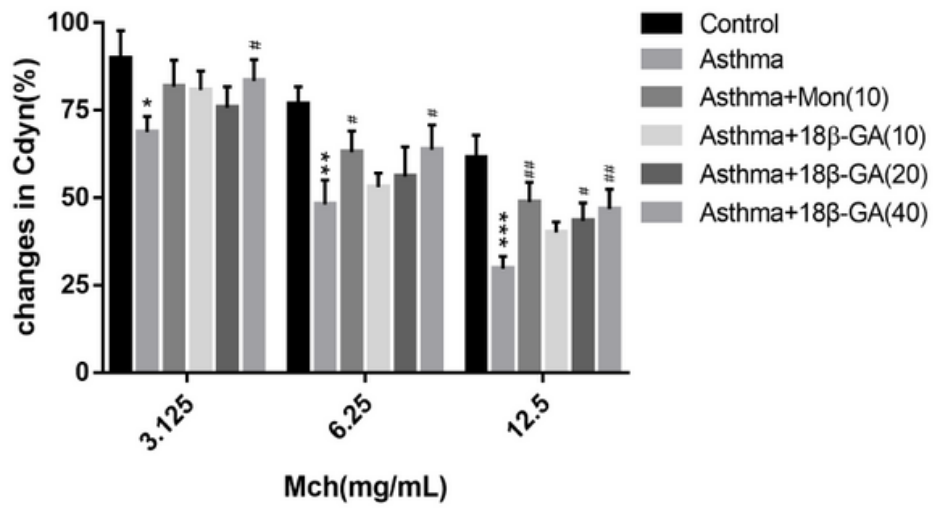

Figure 3

Effect of 18ß-GA treatments on lung function in murine asthmatic model.(a) Increase of lung resistance $(R L \%)$ and (b) decrease of lung dynamic compliance (Cydn\%). Data are shown as the mean \pm SD $(n=6)$. ${ }^{\star} p<0.05,{ }^{* \star} p<0.01,{ }^{\star \star \star} p<0.001$ vs. control group, $\# p<0.05, \# \# p<0.01, \# \# \# p<0.001$ vs. asthma group. 
a

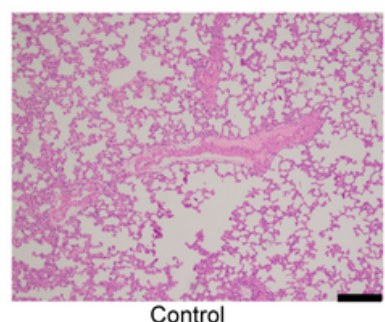

Control

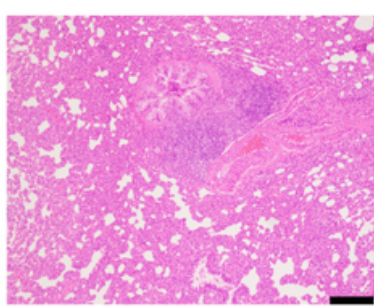

Asthma+18 $\beta-G A(10)$

C

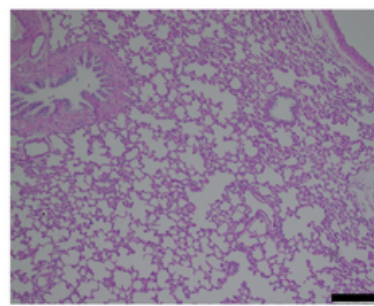

Control

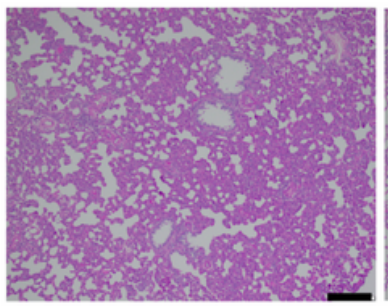

Asthma+18 $\beta-G A(10)$
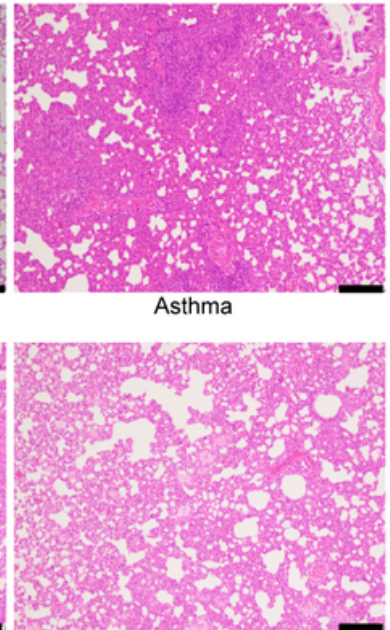

Asthma+18 $\beta-G A(20)$

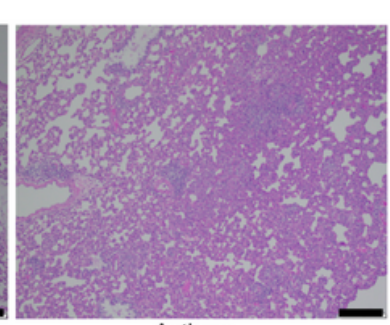

Asthma

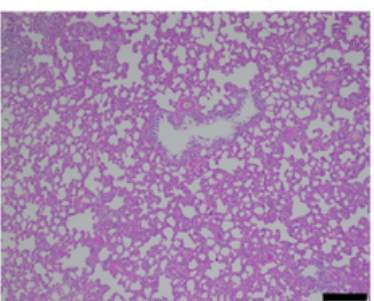

Asthma+18ß-GA(20)
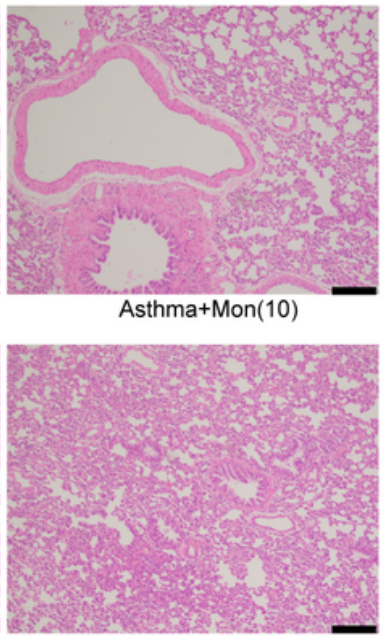

Asthma+18ß-GA(40)

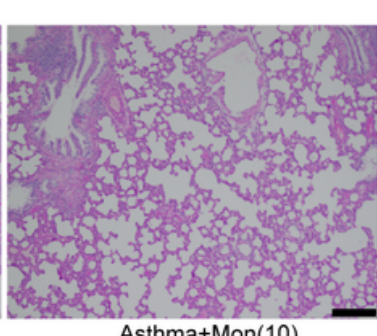

Asthma+Mon(10)

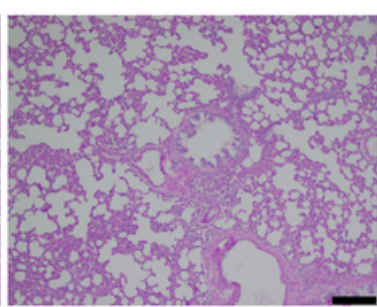

Asthma+18 $\beta-G A(40)$
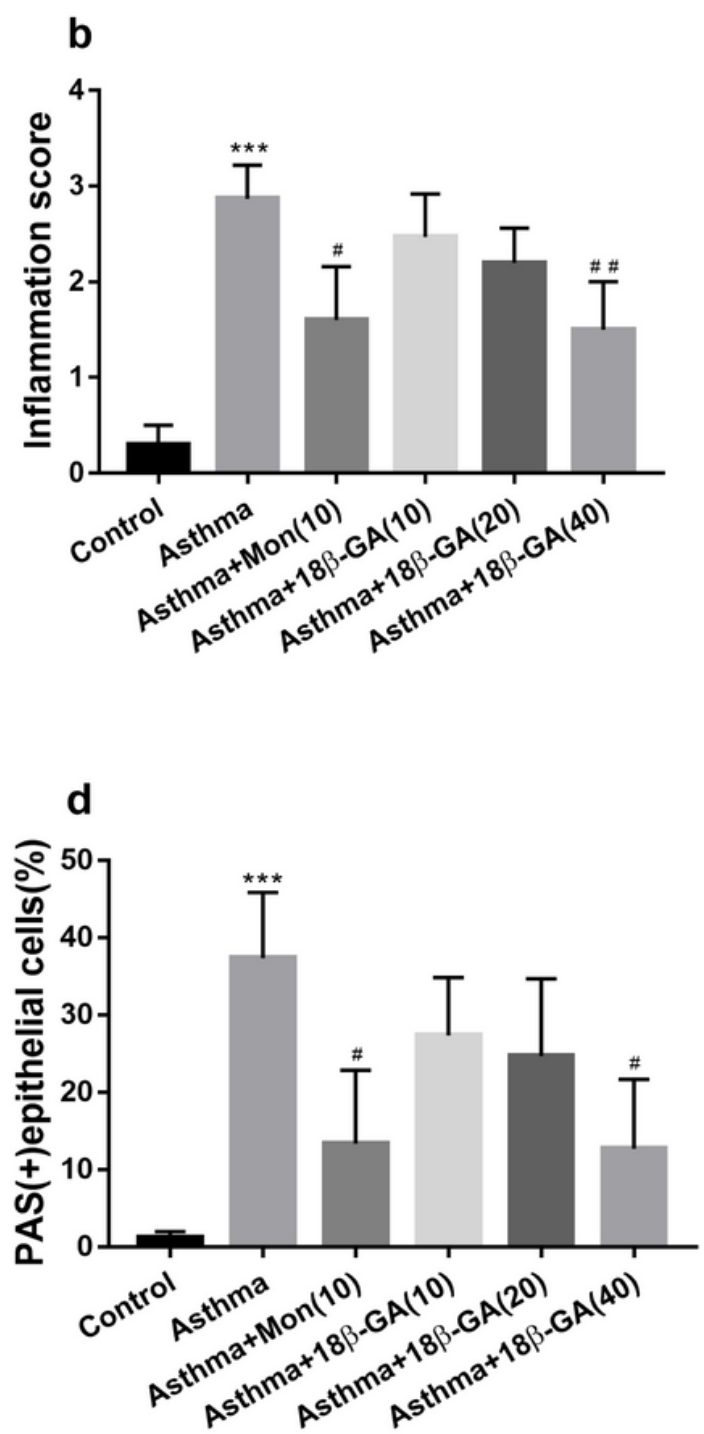

Figure 4

Effects of 18ß-GA on OVA-induced airway inflammation and remodeling in lung tissue. (a) Lung sections were stained with H\&E for measurement of inflammatorycells, (c)PAS for measurement of mucus production around the airways. $\times 100$ magnification, scale bar:100 $\mu \mathrm{m} .(\mathrm{b}, \mathrm{d})$ The column chart of H\&E and PAS were represented the mean $\pm S D(n=6)$. ${ }^{\star} p<0.05$, ${ }^{\star \star} p<0.01$, ${ }^{\star \star \star} p<0.001$ vs. control group, \#p $<$ 0.05 , \#\#p < 0.01, \#\#\#p < 0.001 vs. asthma group. 
a

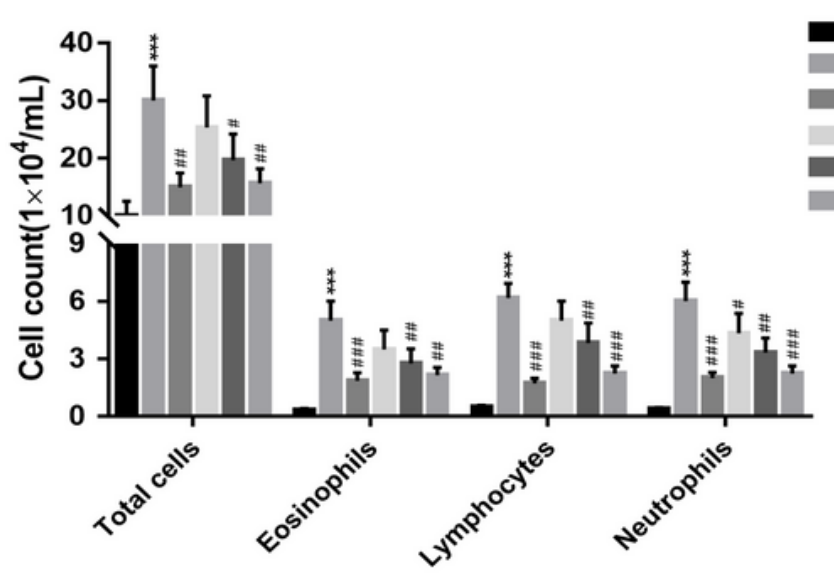

b

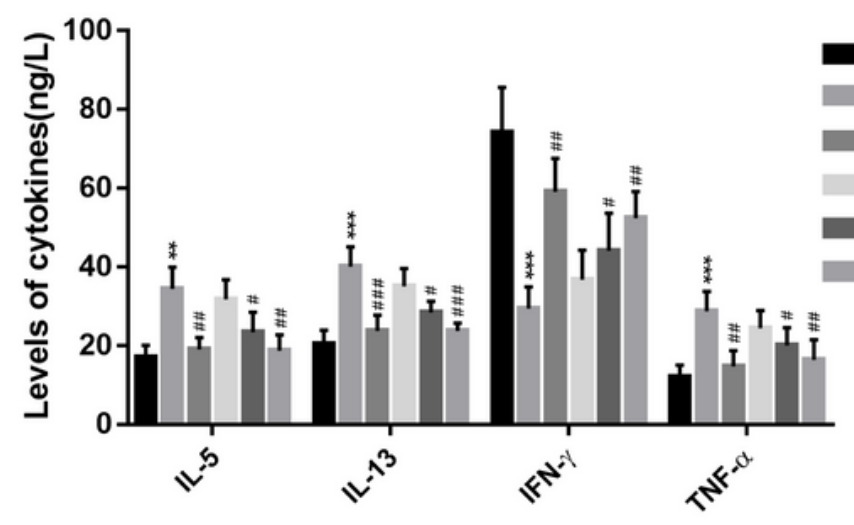

Control

Asthma

Asthma+Mon(10)

Asthma+18ß-GA(10)

Asthma+18ß-GA(20)

Asthma+18ß-GA(40)

C

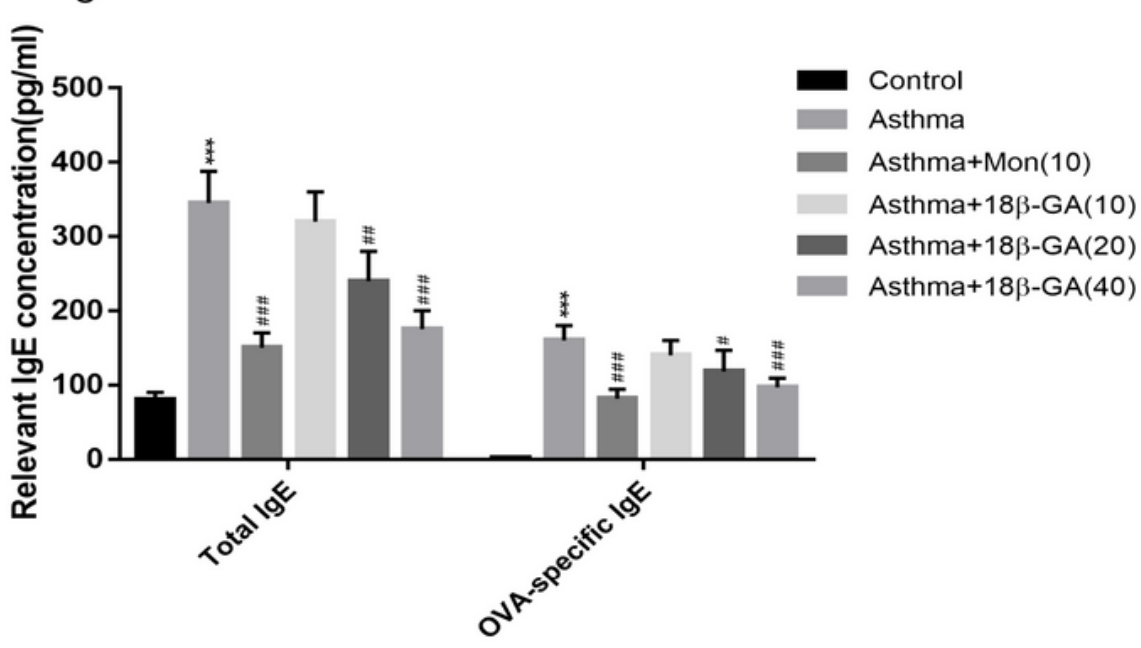

Figure 5

Effect of 18ß-GA on the generation of inflammatory cell infiltration, IL-5, IL-13, IFN- $y$, TNF-a and IgE in BALF. (a) Cell counts in BALF were measured by Diff-Quik staining. (b) The expression levels of IL-5, IL-13, IFN- $\gamma$ and TNF- $\alpha$ in BALF were measured by ELISA. (c) The concentration of IgE in BALF was measured by ELISA. Data are shown as the mean \pm SD $(n=6)$. ${ }^{\star} p<0.05,{ }^{\star *} p<0.01,{ }^{\star \star \star} p<0.001$ vs. control group, \#p $<$ 0.05 , \#\#p < 0.01, \#\#\#p < 0.001 vs. asthma group. 

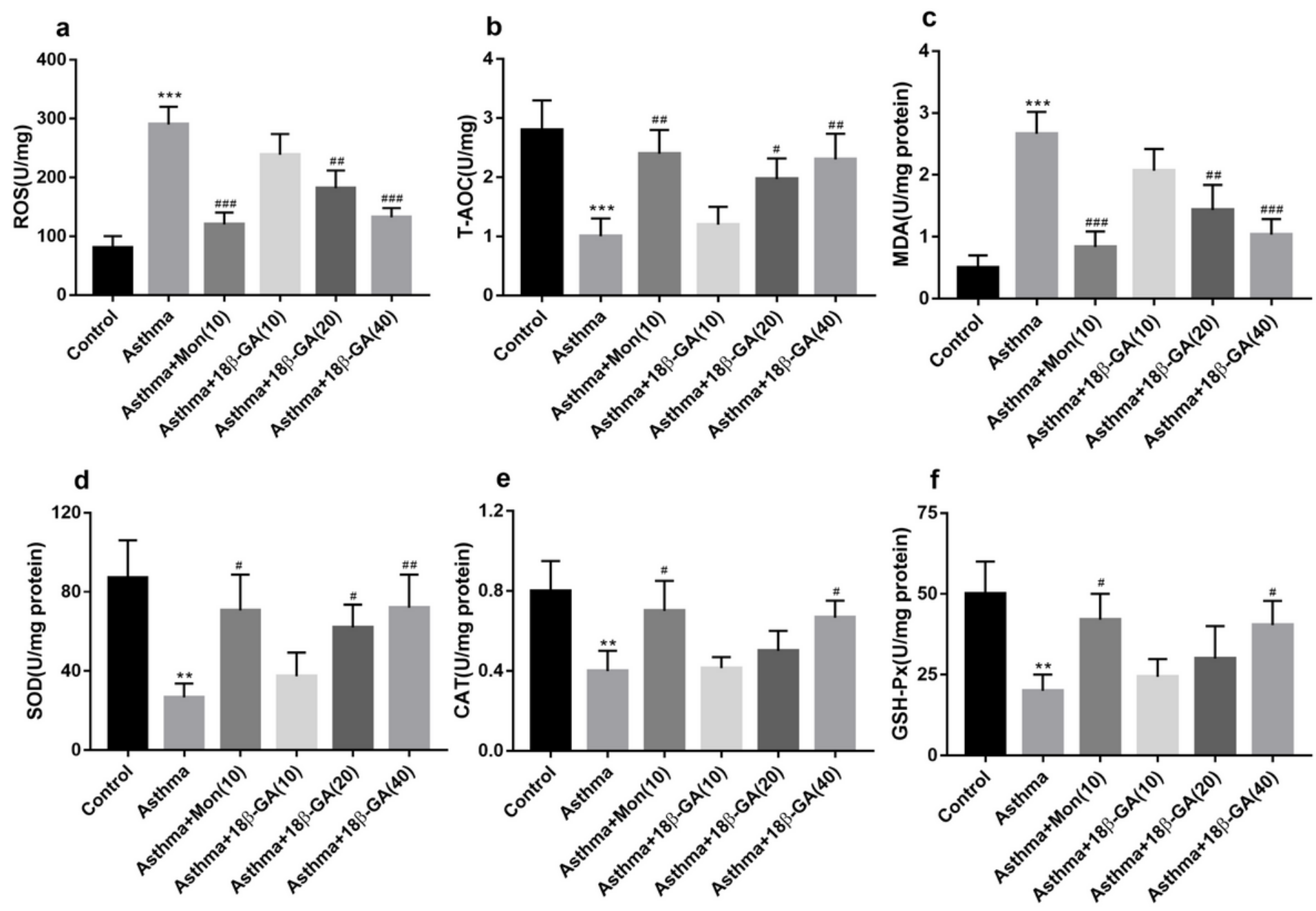

Figure 6

Effects of 18ß-GA on oxidative stress in lung tissue. The levels of (a) ROS, (b) T-AOC, (c) MDA, (d) SOD, (e) CAT and (f)GSH-Px in lung tissues were examined. Data are shown as the mean $\pm S D(n=6)$. ${ }^{*} p<$ $0.05,{ }^{\star \star} p<0.01,{ }^{\star \star \star} p<0.001$ vs. control group, $\# p<0.05$, \#\#p < 0.01, \#\#\#p<0.001 vs. asthma group. TAOC, total antioxidant capacity; MDA, Malondialdehyde; CAT, catalase. 

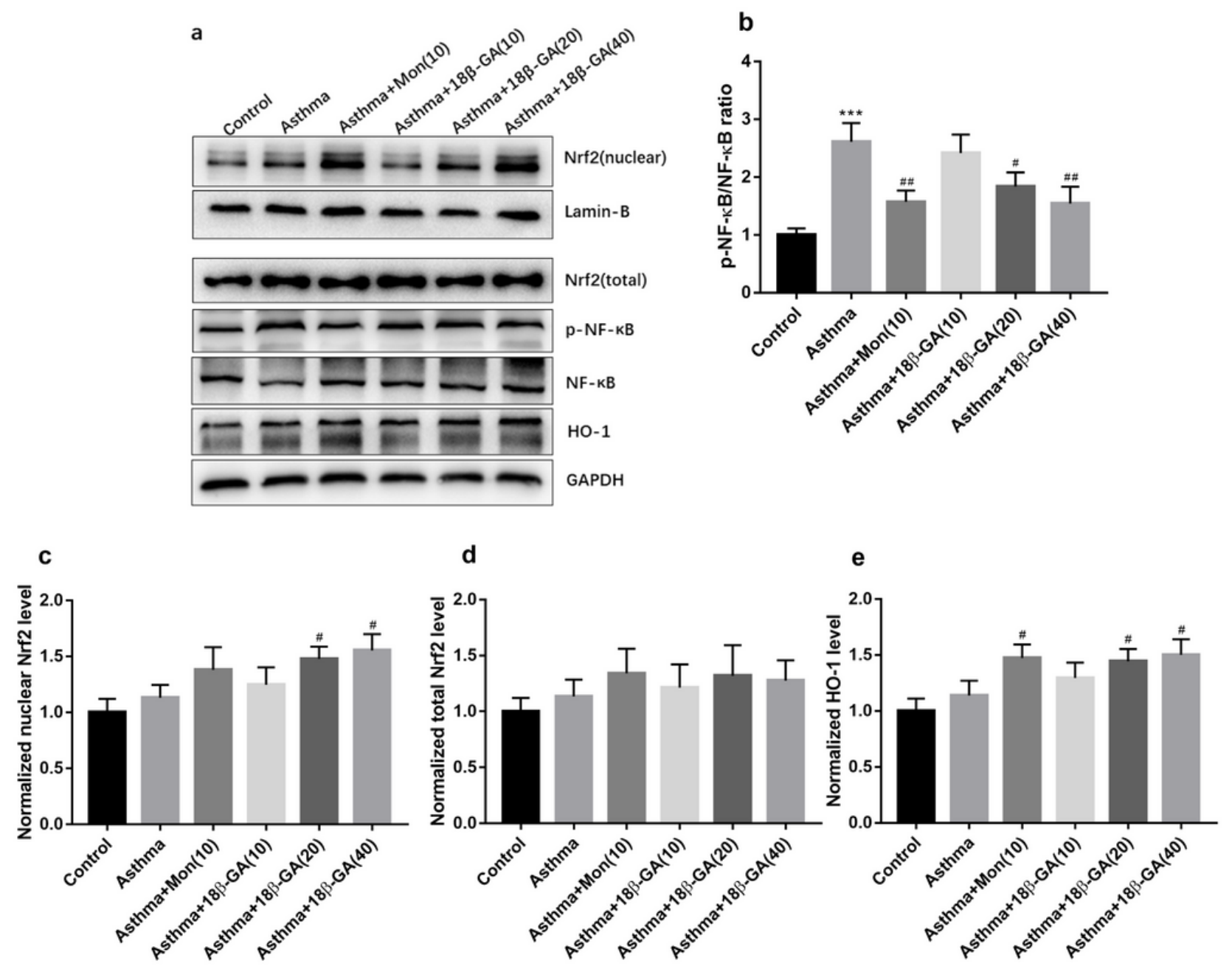

Figure 7

Effect of 18ß-GA on inflammation and oxidative stress signaling pathway. (a)The protein expression of Nrf2, p-NF-KB, NF-KB and HO-1 was determined by western blotting. (b)The relative density quantification of NF-KB. The results were expressed as the ratio of phosphorylated proteins relative to total proteins. (ce) The relative density quantifications of Nrf2(nuclear), Nrf2(total), and HO-1. Data were shown as mean $\pm S D(n=6) .{ }^{\star} p<0.05,{ }^{* \star} p<0.01,{ }^{* \star *} p<0.001$ vs. control group, $\# p<0.05, \# \# p<0.01, \# \# \# p<0.001$ vs. asthma group.

\section{Supplementary Files}

This is a list of supplementary files associated with this preprint. Click to download.

- westernblot1.pdf 